\title{
State Estimation of RC Filters using Unscented Kalman Filter
}

\author{
Amit Kumar Gautam, Sudipta Majumdar
}

\begin{abstract}
This paper implements unscented Kalman filter (UKF) for output voltage estimation of RC low pass filter (LPF) and high pass filter (HPF). At first, the state space model has been obtained using Kirchhoff's current law (KCL). The performance of UKF has been compared with extended Kalman filter $(E K F)$. The simulation results validate the superiority of $U K F$ over $E K F$ as the estimation error is smaller using UKF as compared to the EKF method. As the UKF uses unscented transform (UT) and EKF uses Taylor series expansion for linearization purpose, linearization error is smaller in UKF as compared to EKF method. Also, UKF implementation has the advantage that it does not require Jacobian computation of nonlinear system model.
\end{abstract}

Keywords: Extended Kalman filter, RC filter, state space model, unscented Kalman filter.

\section{INTRODUCTION}

E stimation is fundamental research topic for control system and signal processing. Fast dynamic state estimators are important for proper circuit monitoring. Various state estimation methods have been used in literature. State estimation methods estimate and predict the desired state variables of a dynamic system using noisy measurements. State estimation is helpful for suppression of physical process where states cannot be measured directly or the disturbance have significant role. The Kalman filter (KF), particle filter (PF) and $\mathrm{H}$-infinity filter are some of the state estimation methods in which $\mathrm{KF}$ is the most popular method. KF versions have been applied in various areas such as control system, robotics, etc. [1]

The KF is suitable for linear systems. The variations of KF that can be used for nonlinear systems are: - EKF, Iterated extended Kalman filter (IEKF), UKF, PF and $\mathrm{H}$-infinity filter [2]. These filters use different approaches to handle the nonlinearity. The EKF uses the linearization of both, the transition and measurement functions of the nonlinear system, which involves computation of Jacobian matrices. But, EKF has two important shortcomings. The shortcomings are:-1) Difficulty in determining the Jacobian, 2) These linearization can lead to filter instability, when sufficient small time step intervals are not used. Also, EKF has limitation in prorogating the constraints through the state and covariance calculation. To remove these shortcomings of EKF, Julier et al. [3] and Simon [4] proposed UKF which is based on the fact that it is easier to approximate a probability density function than a nonlinear function.

\footnotetext{
Revised Manuscript Received on June 25, 2020.
}

Amit Kumar Gautam, Senior Research Scholar (SRF), Department of Electronics and Communication Engineering, Delhi Technological University, India.

Sudipta Majumdar, Assistant Professor, Department of Electronics and Communication Engineering Delhi Technological University, India.
UKF uses more accurate approximations to compute multidimensional integral as compared to EKF.

UKF is an advance of KF. A set of sample points called sigma points are used for state distribution in UKF. These sigma points capture the posterior mean and variance of the state distribution. Using a selected set of points, the UKF accurately map the probability distribution of the measurement model. It utilizes the statistical properties of Gaussian variables having nonlinear transformation. The mean and variance are computed using the UT, which avoids the computation of Jacobians. In [5], Ahmeid et al. introduced a new method based on $\mathrm{KF}$ for real time estimation to converter. The KF performance is improved using adaptive tuning methods. Hoffmann et al. [6] used EKF for grid impendence and voltage estimation of power converter of electric network. The noise presented at the connection point has been taken into account. This work also considered the use of EKF in distorted voltage waveform environment. In [7], Nadarajan et al. used EKF for state and parameter estimation of stator winding fault in brushless synchronous generator. The model based method is used and simplified the model for online implementation. In [8], Yazdanian et al. used EKF for parameter estimation of ringdown signal. The method can be implemented for both constant and time varying parameters. In [9], Bogdanski et $a l$. presented identification of vehicle handling dynamics and presented review of UKF, EKF, and PF and showed that all three filters are suitable in real time for online estimation. For this, the paper used simple and efficient model to obtain the independent parameters. Tian et al. [10] proposed UKF based estimation for battery system which uses modified equivalent circuit model. The method presents the low computational cost and improved estimation simultaneously. In [11], Ghahremani et al. implemented UKF in synchronous machine. Simple and effective propagation of probability density function in UKF improved the estimation purpose.

Filters are useful building blocks of various analog integrated circuits. RC filters are useful for various applications. It is used in mixer, samplers and path filters [12]. It is used in chebyshev tracking low pass filter implemented in digital TV tuner integrated circuit. Polyphase $\mathrm{RC}$ filter is used for millimeter wave image reject receivers [13] and in vector sum phase shifter [14]. The active RC filters are useful for various applications. Various realization techniques have been also proposed in literature for active $\mathrm{RC}$ filter. Various approaches are used for continuous line filters topology. The RC active filters have found many applications. In [15],

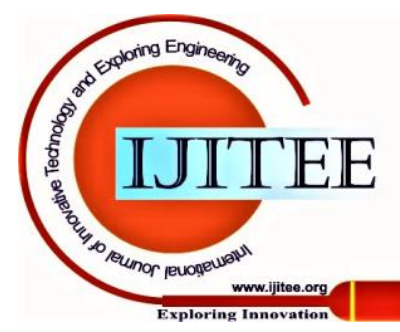


Huang et al. proposed power efficient active RC filters for wireless receivers. Active RC filters are also used in current model transconductance amplifier [16]. It is also used in all pole filters [17]. Active RC filters are also useful for audio frequency bandpass filter [18]. It is also utilized in telephony data communication system, wireless and wireline applications [19]. Conventional LC passive filters are also replaced by active $\mathrm{RC}$ filters in various applications [20]-[22].

The following sections of the paper are as follows: Theory of EKF and UKF has been given in section II. Section III introduces state space representation of second order LPF and HPF circuit using Kirchhoff's current law. Simulation result is given in section IV. Conclusion is given in section $\mathrm{V}$.

\section{ALGORITHM OF EKF AND UKF FOR NONLINEAR SYSTEM}

A nonlinear system is described in discrete time by following equations:

$$
\begin{gathered}
x_{k}=f_{k-1}\left(x_{k-1}, u_{k-1}\right)+w_{k-1} \\
y_{k}=h_{k}\left(x_{k}\right)+v_{k}
\end{gathered}
$$

$x_{k}$ and $y_{k}$ denote as state and measurement vector at time step $k$ respectively. $u_{k}$ is a known input vector. $f_{k}($. and $h_{k}($.$) are nonlinear state and measurement functions$ respectively. $v_{k}$ and $w_{k}$ are the measurement and system noise having zero mean covariance $R_{k}$ and $Q_{k}$ respectively. Consider all assumptions as below:-

$$
\begin{aligned}
& E\left[w_{k}\right]=0, \quad E\left[v_{k}\right]=0, \quad E\left[w_{k}, w_{j}^{T}\right]=0 \forall k \neq j, \\
& E\left[w_{k}, w_{k}^{T}\right]=Q_{k}, E\left[v_{k}, v_{k}^{T}\right]=R_{k}, E\left[v_{k}, x_{0}^{T}\right]=0 \quad k, \\
& E\left[w_{k}, x_{0}^{T}\right]=0 \forall k, E\left[w_{k}, v_{j}^{T}\right]=0 \forall k \& j .
\end{aligned}
$$

\section{A. Extended Kalman Filter}

EKF uses the linearization of the nonlinear function via Taylor's series as:

$$
\begin{gathered}
x_{k}=f_{k-1}\left(\hat{x}_{k-1 \mid k-1}, u_{k-1}\right)+F_{k-1} \tilde{x}_{k-1}+ \\
\text { Higher order terms } \\
y_{k}=h_{k}\left(f_{k-1}\left(\hat{x}_{k-1 \mid k-1}, u_{k-1}\right)\right)+H_{k} \tilde{x}_{k-1}+
\end{gathered}
$$$$
\text { Higher order terms }
$$

where

$$
F_{k}=\left.\frac{\partial f_{i}\left(x, u_{k}\right)}{\partial x_{j}}\right|_{x=\hat{x}_{k \mid k-1}}, H_{k}=\left.\frac{\partial h_{i}\left(x, u_{k}\right)}{\partial x_{j}}\right|_{x=\hat{x}_{k \mid k}}
$$

The following are the EKF steps:

1) Initialization: Initialize $\hat{x}_{0}, P_{0}, Q_{k}$ and $R_{k}$.

2) Prediction of states: Compute state prediction and covariance as:

$$
\begin{gathered}
\hat{x}_{k \mid k-1}=f_{k-1}\left(\hat{x}_{k-1 \mid k-1}, u_{k-1}\right) \\
P_{k \mid k-1}=F_{k} P_{k-1 \mid k-1} F_{k}^{T}+Q_{k-1}
\end{gathered}
$$

\section{3) Measurement update:}

a) Calculate Kalman gain as:

$$
K_{k}=P_{k \mid k-1} H_{k}^{T}\left(H_{k} P_{k \mid k-1} H_{k}^{T}+R_{k}\right)^{-1}
$$

b) Compute updated state as:

$$
\hat{x}_{k \mid k}=\hat{x}_{k \mid k-1}+K_{k}\left(y_{k}-h_{k}\left(\hat{x}_{k \mid k-1}\right)\right)
$$

c) Compute update covariance as:

$$
P_{k \mid k}=\left(I-K_{k} H_{k}\right) P_{k \mid k-1}
$$

where $k \mid k-1$ and $k \mid k$ are a prior and a post state estimation.

\section{B. Unscented Kalman Filter}

The UKF is based on statistical approach, which overcomes the limitations of EKF. These are:- 1) poor approximation approach of the first order linearization, and 2) To avoid Jacobian computation. The mean and variance are computed using UT. The steps for UKF algorithm are as follows:

1) Prediction Step:-

a) Initialize $\hat{x}_{0}$ and $P_{0}$.

b) Calculate sigma points for $i=2 n+1$ as:-

$X_{i, k-1}=\left\{\hat{x}_{k-1}, \hat{x}_{k-1}+\left(\gamma \sqrt{P_{k-1}}\right)_{i}, \hat{x}_{k-1}-\left(\gamma \sqrt{P_{k-1}}\right)_{i}\right\}$

where $\gamma=\sqrt{n+\lambda} . n$ and $\lambda$ denote state number and scaling constant.

c) Update time propagation using transformed sigma points as:-

$$
\mathcal{X}_{i, k \mid k-1}=f\left(\mathcal{X}_{i, k-1}, u_{k-1}\right)
$$

d) Calculate priori state and covariance error as:-

$$
\hat{x}_{k \mid k-1}=\sum_{i=0}^{2 n} W_{i}^{(m)} \mathcal{X}_{i, k \mid k-1}
$$

$$
\hat{P}_{k \mid k 1}={ }_{i=0}^{2 n} W_{i}^{(c)}\left(X_{i, k \mid k 1} \quad \hat{x}_{k \mid k 1}\right)\left(X_{i, k \mid k 1} \quad \hat{x}_{k \mid k 1}\right)^{T}+Q_{k 1}
$$

where

$$
\begin{gathered}
W_{0}^{(m)}=\frac{\lambda}{\lambda+n}, W_{0}^{(c)}=\frac{\lambda}{\lambda+n}+\left(1-\alpha^{2}+\beta\right), \\
W_{i}^{(m)}=W_{i}^{(c)}=\frac{\lambda}{2(\lambda+n)}, \text { for } i=1, \ldots, 2 n
\end{gathered}
$$

Here, $\alpha$ and $\beta$ indicate tuning parameter and positive weighing parameter

2) Measurement Update:- It consist of following steps:-

a) Update sigma points to predict the output using

$$
\begin{aligned}
& Y_{i, k \mid k-1}=h\left(\mathcal{X}_{i, k \mid k-1}, u_{k}\right) \\
& \hat{y}_{k \mid k-1}=\sum_{i=0}^{2 n} W_{i}^{(m)} Y_{i, k \mid k-1}
\end{aligned}
$$

b) Kalman gain $K_{k}$ is as: 


$$
\begin{aligned}
K_{k}= & {\left[\sum_{i=0}^{2 n} W_{i}^{(c)}\left(Y_{i, k \mid k-1}-\hat{y}_{k \mid k-1}\right)\left(Y_{i, k \mid k-1}-\hat{y}_{k \mid k-1}\right)^{T}+R_{k}\right] } \\
& \times\left[\sum_{i=0}^{2 n} W_{i}^{(c)}\left(X_{i, k \mid k-1}-\hat{x}_{k \mid k-1}\right)\left(Y_{i, k \mid k-1}-\hat{y}_{k \mid k-1}\right)^{T}\right]^{-1}
\end{aligned}
$$

c) Posteriori state and covariance error are calculated as:-

$$
\begin{gathered}
\hat{x}_{k}=\hat{x}_{k \mid k 1}+K_{k}\left(\begin{array}{ll}
y_{k} & \hat{y}_{k \mid k 1}
\end{array}\right) \\
P_{k}=P_{k \mid k-1}-K_{k} P_{y_{k} y_{k}} K_{k}^{T}
\end{gathered}
$$

\section{MATHEMATICAL MODELING OF HIGHER ORDER RC CIRCUIT}

Second order RC LPF shown in Fig. 1. It consists of series parallel combination of resistor $(\mathrm{R})$ and capacitor $(\mathrm{C})$. Input sinusoidal voltage $u_{1}(t)$ is applied to the circuit. Capacitor voltages across $C_{1}$ and $C_{2}$ are $V_{c_{1}}$ and $V_{c_{2}}$ respectively.

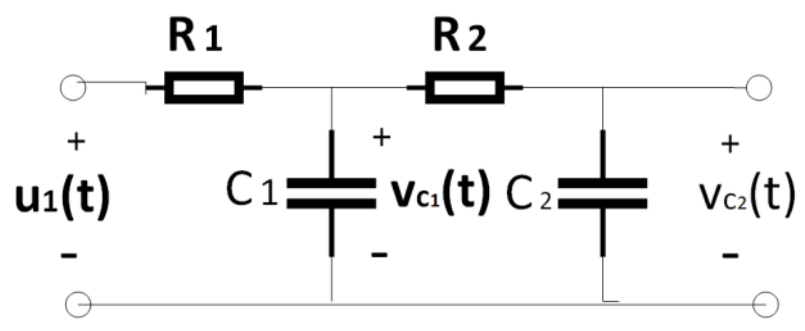

Fig. 1.Circuit diagram of RC LPF.

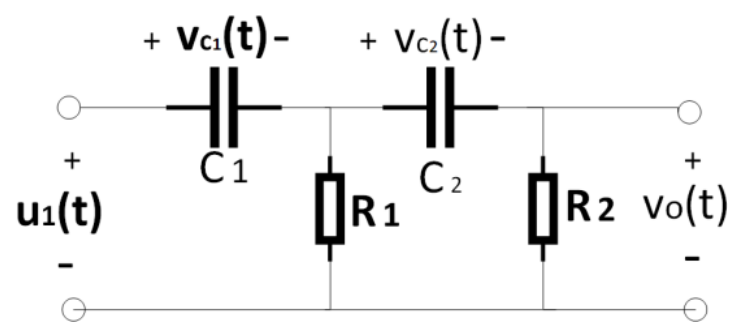

Fig. 2.Circuit diagram of RC HPF.

The state space model for the RC LPF circuit is obtained using KCL as:

$$
\begin{gathered}
\frac{v_{c_{1}}(t)-u_{1}(t)}{R_{1}}+\frac{v_{c_{1}}(t)-v_{c_{2}}}{R_{2}}+C_{1} \frac{d v_{c_{1}}}{d t}=0 \\
\frac{v_{c_{2}}(t)-v_{c_{1}}}{R_{2}}+C_{2} \frac{d v_{c_{2}}}{d t}=0
\end{gathered}
$$

Representing (20)-(21) as state space model, we have

$$
\frac{d}{d t} X=A X+B U
$$

$$
A=\left[\begin{array}{cc}
-\frac{1}{C_{1}}\left(\frac{1}{R_{1}}+\frac{1}{R_{2}}\right) & \frac{1}{R_{1} C_{1}} \\
\frac{1}{R_{2} C_{2}} & -\frac{1}{R_{2} C_{2}}
\end{array}\right] \text { and } B=\left[\begin{array}{cc}
\frac{1}{R_{1} C_{1}} & 0
\end{array}\right]^{T}
$$

$Y$ is the measurement model given as:-

$$
Y=H X+V
$$

where $H$ denotes the measurement vector as:-

$$
H=\left[\begin{array}{ll}
1 & 0 \\
0 & 1
\end{array}\right]^{T} \text { and } D=0 \text {. }
$$

The state model in (22) and (23) can be discretized using first order exponential method. In general, the discretized state space model are represented as

$$
\begin{gathered}
X_{k+1}=A_{k} X_{k}+B_{k} v_{s_{k}}+w_{k} \\
Y_{k}=H_{k} X_{k}+v_{k}
\end{gathered}
$$

Here, the discrete parameters $A_{k}, B_{k}$ and $H_{k}$ have been obtained using Euler-Maruyama method and using $t=k T_{s}$, $k=1,2,3,4 \ldots$ where $T_{s}$ is sampling time.

$$
\begin{gathered}
A_{k}=\left[\begin{array}{ccc}
1 & \frac{T_{s}}{C_{1}}\left(\frac{1}{R_{1}}+\frac{1}{R_{2}}\right) & \frac{T_{s}}{R_{1} C_{1}} \\
\frac{T_{s}}{R_{2} C_{2}} & 1 & \frac{T_{s}}{R_{2} C_{2}}
\end{array}\right], \\
B_{k}=\left[\begin{array}{cc}
\frac{T_{s}}{R_{1} C_{1}} & 0
\end{array}\right]^{T}, H_{k}=\left[\begin{array}{ll}
1 & 0 \\
0 & 1
\end{array}\right]^{T}
\end{gathered}
$$

Similarly, state space model for RC HPF shown in Fig. 2 can be represented as

$$
\begin{aligned}
\frac{d}{d t}\left[\begin{array}{l}
v_{c_{1}}(t) \\
v_{c_{2}}(t)
\end{array}\right] & =\left[\begin{array}{cc}
-\frac{1}{C_{1}}\left(\frac{1}{R_{1}}+\frac{1}{R_{2}}\right) & \frac{1}{R_{2} C_{1}} \\
\frac{1}{R_{2} C_{2}} & -\frac{1}{R_{2} C_{2}}
\end{array}\right]\left[\begin{array}{l}
v_{c_{1}}(t) \\
v_{c_{2}}(t)
\end{array}\right] \\
& +\left[\begin{array}{c}
\frac{1}{C_{1}}\left(\frac{1}{R_{1}}+\frac{1}{R_{2}}\right) \\
\frac{1}{R_{2} C_{2}}
\end{array}\right] u_{2}(t) \\
y & =\left[\begin{array}{ll}
-1 & -1]
\end{array}\right]\left[\begin{array}{l}
v_{c_{1}}(t) \\
v_{c_{2}}(t)
\end{array}\right]+u_{2}(t)
\end{aligned}
$$

where 
where the matrices $A_{k}, B_{k}$ and $H_{k}$ for RC HPF are obtained by discretizing equations (27)-(28). They are:

$$
\begin{gathered}
A_{k}=\left[\begin{array}{ccc}
1 \frac{T_{s}}{C_{1}}\left(\frac{1}{R_{1}}+\frac{1}{R_{2}}\right) & \frac{T_{s}}{R_{2} C_{1}} \\
\frac{T_{s}}{R_{2} C_{2}} & 1 \frac{T_{s}}{R_{2} C_{2}}
\end{array}\right], \\
B_{k}=\left[\begin{array}{cc}
\frac{T_{s}}{R_{1} C_{1}} & 0
\end{array}\right]^{T}, H_{k}=\left[\begin{array}{ll}
1 & 0 \\
0 & 1
\end{array}\right]^{T}
\end{gathered}
$$

\section{SIMULATION RESULTS}

The estimation of output voltage of RC LPF and HPF have been performed in MATLAB software. The estimation have been performed for two different cases:- (i) noiseless input and (ii) noisy input. The applied sinusoidal input with maximum amplitude of $10 \mathrm{~V}$ and frequency $0.04 \mathrm{~Hz}$ is shown in Fig. 3. The white Gaussian noise of zero mean and different variances has been used as noisy input for estimation purpose. The PSPICE simulated values have been taken as actual value. The output voltages of LPF and HPF circuit using UKF and EKF under different noisy inputs have been shown in Fig. 4 to 9. Fig. 10 to 15 presents the error comparison of EKF and UKF for different noise values. Table I and II show the comparison of root mean square error (RMSE) and signal to noise ratio (SNR) for both circuit using EKF and UKF methods.

$$
\begin{gathered}
S N R=10 \log _{10}\left[\frac{\sum_{i=1}^{n}\left(\hat{y}_{i}\right)^{2}}{\sum_{i=1}^{n}\left(\hat{y}_{i}-y_{i}\right)^{2}}\right] \\
R M S E=\sqrt{\frac{\sum_{i=1}^{n}\left(\hat{y}_{i}-y_{i}\right)^{2}}{n}}
\end{gathered}
$$

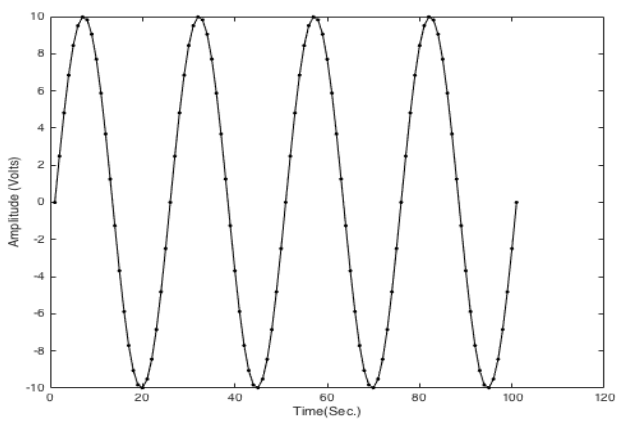

Fig. 3.Input sinusoidal voltage.

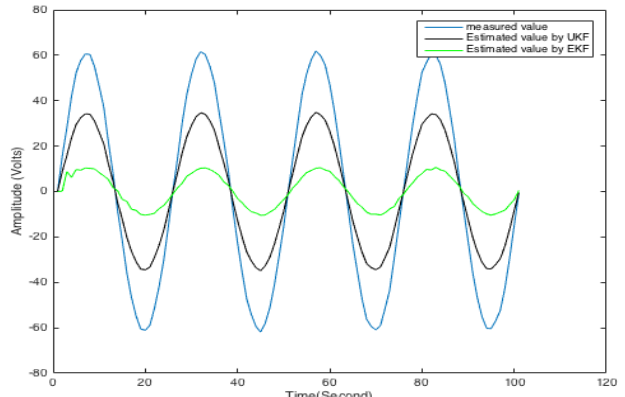

Fig. 4.Output voltage estimation of LPF using EKF and UKF methods for Gaussian noisy input $\left({ }^{2}=0.1\right)$

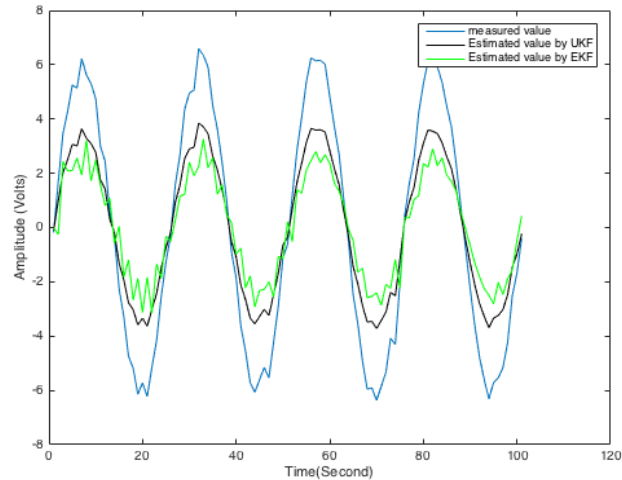

Fig. 5.Output voltage estimation of LPF using EKF and UKF methods for Gaussian noisy input $\left({ }^{2}=0.5\right.$ )

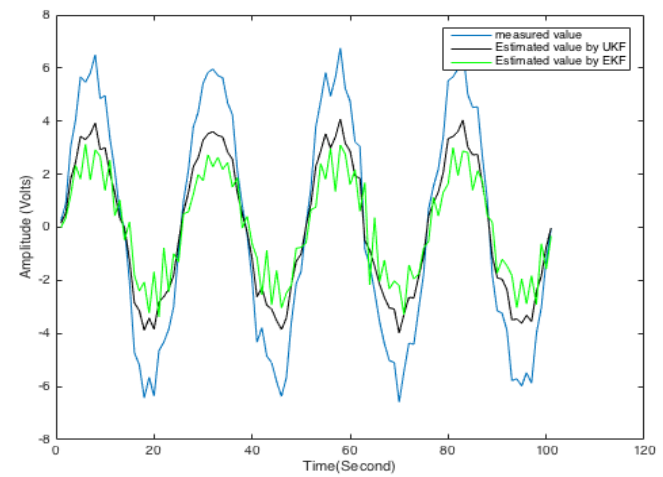

Fig. 6.Output voltage estimation of LPF using EKF and UKF methods for Gaussian noisy input $\left({ }^{2}=1.0\right.$ )

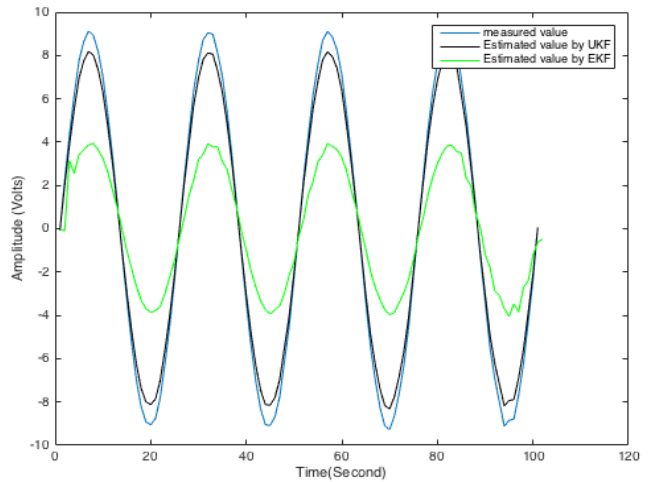

Fig. 7.Output voltage estimation of HPF using EKF and UKF methods for Gaussian noisy input $\left({ }^{2}=0.1\right)$

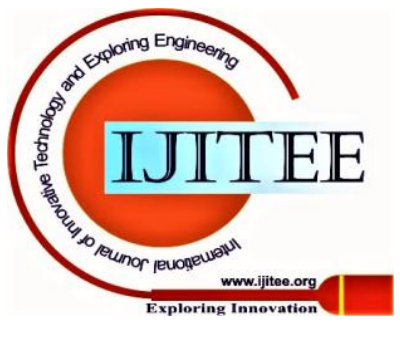




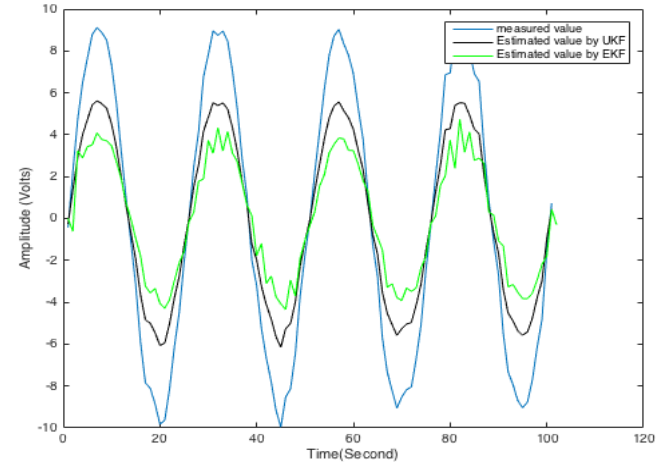

Fig. 8.Output voltage estimation of HPF using EKF and UKF methods for Gaussian noisy input $\left({ }^{2}=0.5\right)$

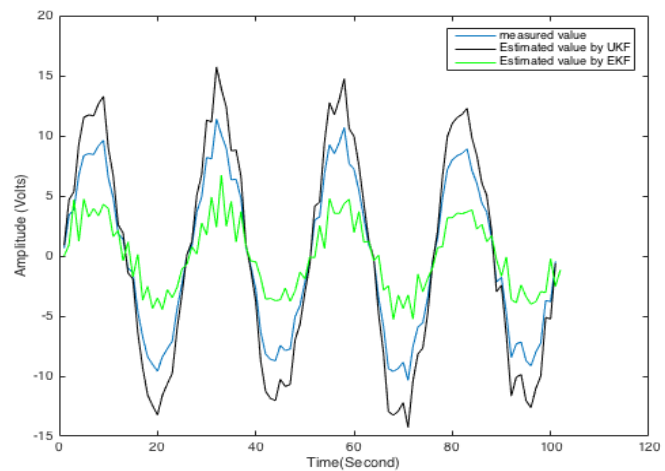

Fig. 9.Output voltage estimation of HPF using EKF and UKF methods for Gaussian noisy input $\left({ }^{2}=1.0\right)$

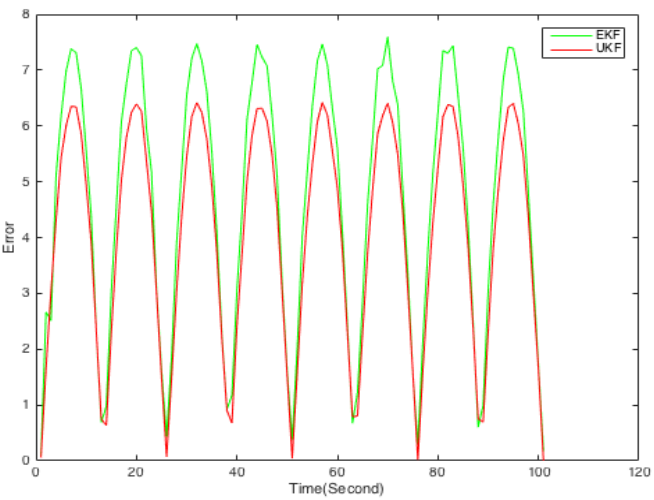

Fig. 10. RMSE of LPF using EKF and UKF methods for Gaussian noisy input $\left({ }^{2}=0.1\right)$

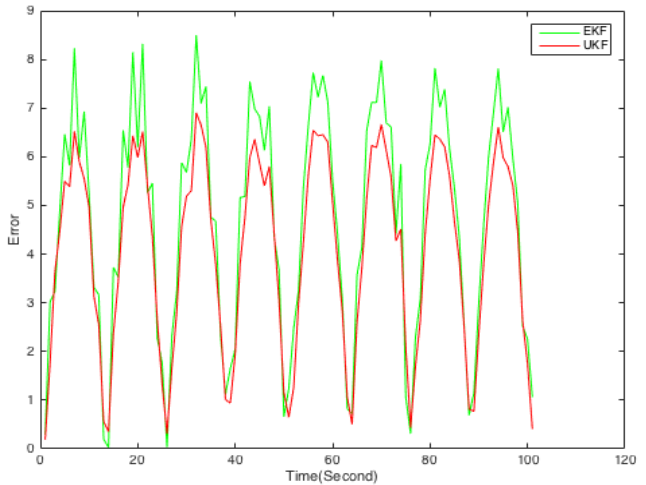

Fig. 11. RMSE of LPF using EKF and UKF methods for Gaussian noisy input $\left({ }^{2}=0.5\right)$

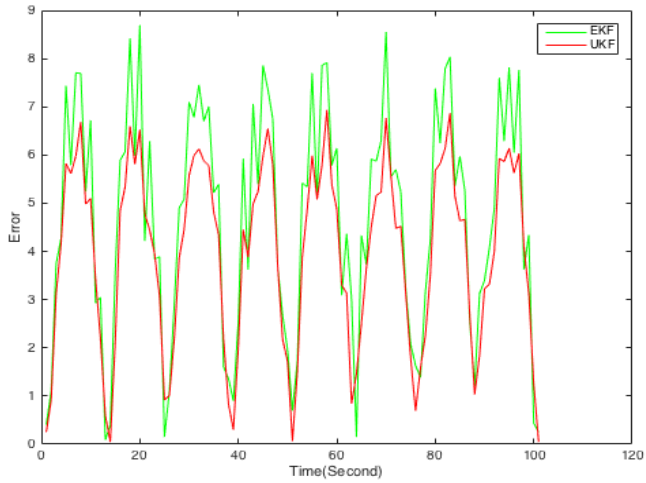

Fig. 12. RMSE of LPF using EKF and UKF methods for Gaussian noisy input $\left({ }^{2}=1.0\right)$

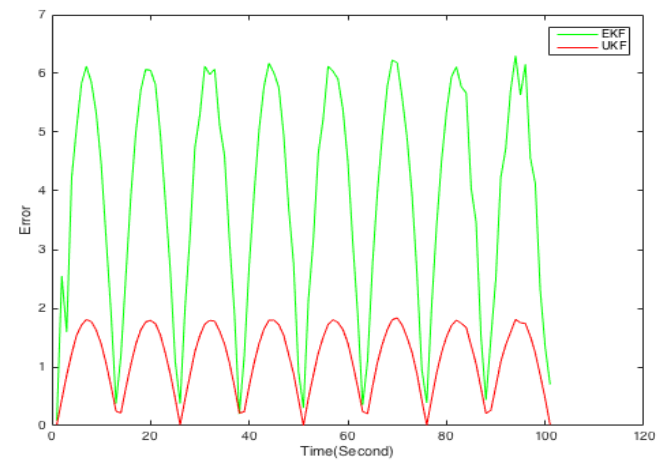

Fig. 13. RMSE of HPF using EKF and UKF methods for Gaussian noisy input $\left({ }^{2}=0.1\right)$

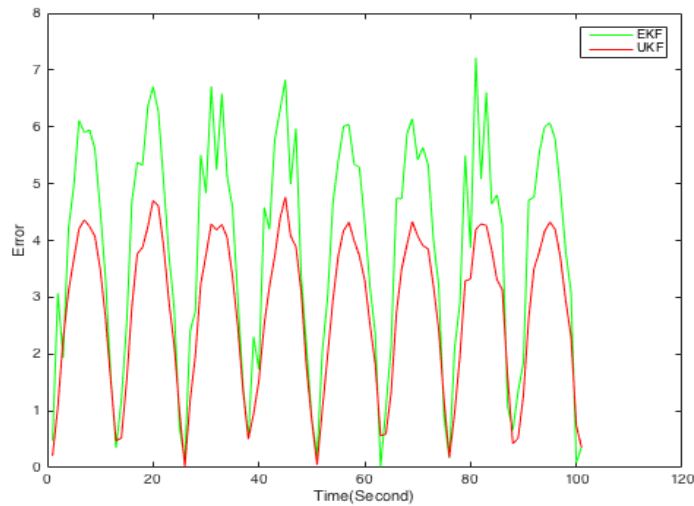

Fig. 14. RMSE of HPF using EKF and UKF methods for Gaussian noisy input $\left({ }^{2}=0.5\right)$

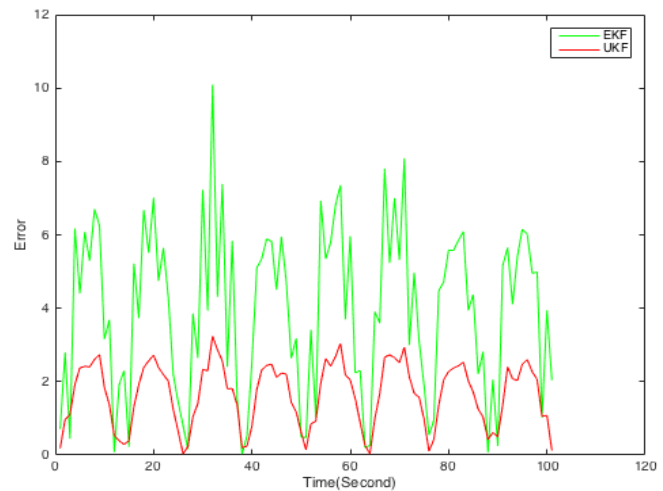

Fig. 15. RMSE of HPF using EKF and UKF methods for Gaussian noisy input $\left({ }^{2}=1.0\right)$

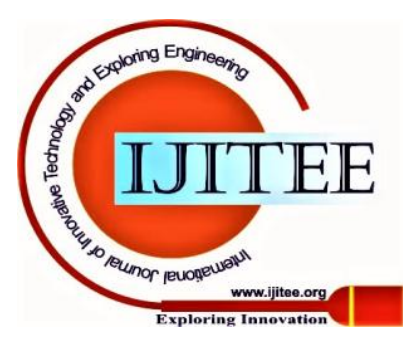


Table I: Performance of different methods for LPF

\begin{tabular}{|l|c|c|c|}
\hline \multicolumn{1}{|c|}{ Signal } & Parameter & EKF & UKF \\
\hline $\begin{array}{l}\text { Noisy input } \\
\text { signal } \\
\left(\sigma^{2}=0.1\right)\end{array}$ & SNR(dB) & 24.44 & 40.55 \\
\cline { 2 - 4 } & RMSE & 0.2842 & 0.0402 \\
\hline $\begin{array}{l}\text { Noisy input } \\
\text { signal } \\
\left(\sigma^{2}=0.5\right)\end{array}$ & SNR(dB) & 11.17 & 31.56 \\
\cline { 2 - 4 } & RMSE & 0.5803 & 0.0991 \\
\hline $\begin{array}{l}\text { Noisy input } \\
\text { signal } \\
\left(\sigma^{2}=1.0\right)\end{array}$ & SNR(dB) & 7.57 & 23.66 \\
\cline { 2 - 4 } & RMSE & 1.4794 & 1.2746 \\
\hline
\end{tabular}

Table II: Performance of different methods for HPF

\begin{tabular}{|l|c|c|c|}
\hline \multicolumn{1}{|c|}{ Signal } & Parameter & EKF & UKF \\
\hline $\begin{array}{l}\text { Noisy input } \\
\text { signal } \\
\left(\sigma^{2}=0.1\right)\end{array}$ & SNR(dB) & 28.57 & 40.12 \\
\cline { 2 - 4 } & RMSE & 0.1588 & 0.0457 \\
\hline $\begin{array}{l}\text { Noisy input } \\
\text { signal } \\
\left(\sigma^{2}=0.5\right)\end{array}$ & SNR(dB) & 14.46 & 26.15 \\
\cline { 2 - 4 } & RMSE & 1.0521 & 0.1867 \\
\hline $\begin{array}{l}\text { Noisy input } \\
\text { signal } \\
\left(\sigma^{2}=1.0\right)\end{array}$ & SNR(dB) & 8.96 & 20.99 \\
\cline { 2 - 4 } & RMSE & 1.810 & 0.2175 \\
\hline
\end{tabular}

\section{CONCLUSIONS}

The state estimation of RC LPF and HPF using UKF is presented in this paper and compared with EKF method. Simulation results show the better closeness of estimated capacitor voltage and diode current with PSPICE simulated values as compared to the EKF method due to smaller linearization error of UKF. Also, the SNR value of UKF is better than EKF. UKF presents smaller RMSE as compared to EKF as UKF is accurate to the third order for any nonlinearity.

\section{REFERENCES}

1. F. Auger, M. Hilairet, J. M. Guerrero, E. Monmasson, T. Orlowska-Kowalska and S. Katsura, "Industrial applications of the Kalman filter: A review," IEEE Trans. on Industrial Electronics, vol. 60, no. 12, 2013, pp. 5458-5471.

2. P. Stano, Z. Lendek and J. Braaksma, R. Babuška, C. D. Keizer and A. J. D. Dekker, "Parametric Bayesian filters for nonlinear stochastic dynamical systems: A survey," IEEE Trans. on cybernetics, vol. 43, no. 6, 2013, pp. 1607-1624.

3. S. J. Julier, J. K. Uhlmann and H. F. Durrant-Whyte, "A new approach for filtering nonlinear systems," In American Control Conference, Proceedings of the 1995, vol. 3, 1995, pp. 1628-1632.

4. D. Simon, "Optimal state estimation: Kalman, $\mathrm{H}$ infinity, and nonlinear approaches,"John Wiley and Sons, 2006.

5. M. Ahmeid, M. Armstrong, S. Gadoue, M. Al-Greer, P. Missailidis, "Real-Time Parameter Estimation of DC-DC Converters Using a Self-Tuned Kalman Filter," IEEE Trans. on Power Electronics, vol. 32, no. 7, 2017, pp. 5666-5674.

6. N. Hoffmann and F. W. Fuchs, "Minimal invasive equivalent grid impedance estimation in inductive-resistive power networks using extended Kalman filter," IEEE Trans. on Power Electronics, vol. 29, no. 2, 2014, pp. 631-641.

7. S. Nadarajan, S. K. Panda, B. Bhangu and A. K. Gupta, "Online model-based condition monitoring for brushless wound-field synchronous generator to detect and diagnose stator windings turn-to-turn shorts using extended Kalman filter," IEEE Trans. on Industrial Electronics, vol. 63, no. 5, 2016, pp. 3228-3241.

8. M. Yazdanian, A. Mehrizi-Sani and M. Mojiri, "Estimation of electromechanical oscillation parameters using an extended Kalman filter," IEEE Trans. on Power Systems, vol. 30, no. 6, 2015, pp. 2994-3002.

9. K. Bogdanski and M. C. Best, "Kalman and particle filtering methods for full vehicle and tyre identification," Vehicle System Dynamics, vol. 56, no.5, 2018, pp. 769-90.

10. Y. Tian, B. Xia, W. Sun, Z. Xu and W. Zheng, "A modified model based state of charge estimation of power lithium-ion batteries using unscented Kalman filter," Journal of power sources, vol. 270, 2014, pp. 619-626.

11. E. Ghahremani, and I. Kamwa, "Online state estimation of a synchronous generator using unscented Kalman filter from phasor measurements units," IEEE Trans. on Energy Conversion, vol. 26, no. 4, 2011, pp. 1099-1108.

12. S. Pavan and E. Klumperink, "Simplified Unified Analysis of Switched-RC Passive Mixers, Samplers, and N-Path Filters Using the Adjoint Network," IEEE trans. on circuits and systems I: regular papers, vol. 64, no. 10, 2017, pp. 2714-2725.

13. L. E. Milner, L. T. Hall, J. T. Harvey, M. E. Parker, "A polyphase RC filter implemented in ring configuration for a SiGe millimetre-wave receiver," In IEEE 2nd Australian Microwave Symposium (AMS), 2016, pp. 15-16.

14. M. Momeni and M. Moezzi, "A Low Loss and Area Efficient RC Passive Poly Phase Filter for Monolithic GHz Vector-Sum Circuits," IEEE Trans. on Circuits and Systems II: Express Briefs, vol. 66, no. 7 , 2018, pp. 1134-1138.

15. L. Ye, C. Shi, H. Liao, R. Huang, Y. Wang, "Highly power-efficient active-RC filters with wide bandwidth-range using low-gain push-pull opamps," IEEE Trans. on Circuits and Systems I: Regular Papers, vol. 60, no. 1, 2012, pp. 95-107.

16. D. V. Kamat, P. A. Mohan, K. G. Prabhu, "Current-mode operational transconductance amplifier-capacitor biquad filter structures based on Tarmy-Ghausi Active-RC filter and second-order digital all-pass filters," IET circuits, devices \& systems, vol. 4, no. 4, 2010, pp. 346-364.

17. G. S. Moschytz, "Low-sensitivity, low-power active-RC all pole filters using impedance tapering," IEEE Trans. on Circuits and Systems II: Analog and Digital Signal Processing, vol. 46, no. 8, 1999, pp. 1009-1026.

18. D. G. Haigh and R. Jeffers, "The design of an audio-frequency active RC band-pass filter for a specific engineering requirement," Radio and Electronic Engineer, vol. 42, no. 8, 1972, pp. 373-380.

19. H. Amir-Aslanzadeh, E. J. Pankratz, E. Sanchez-Sinencio, "A 1-V+ 31 $\mathrm{dBm}$ IIP3, reconfigurable, continuously tunable, power-adjustable active-RC LPF," IEEE Journal of Solid-State Circuits, vol.44, no. 2, 2009, pp. 495-508.

20. Y. Ishibashi, "Active RC filter based on simulation of dissipative LC filters," IEE Proceedings-Circuits, Devices and Systems, vol. 141, vol. 2, 1994, pp. 101-104.

21. S. Pavan and E. Klumperink, "Generalized Analysis of High-Order Switch-RC N-Path Mixers/Filters Using the Adjoint Network," IEEE Transactions on Circuits and Systems I: Regular Papers, 2018.

22. D. Li, D. Basak, Y. Zhang, Z. Fu, K. P. Pun, "Improving Power Efficiency for Active-RC Delta-Sigma Modulators Using Passive-RC Low-Pass Filter in Feedback," IEEE Transactions on Circuits and Systems II: Express Briefs, 2017, pp. 1549-7747.

\section{AUTHORS PROFILE}

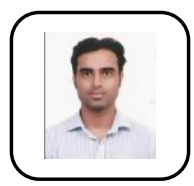

Amit Kumar Gautam was born in Uttar Pradesh, India in the year of 1987. He got B.Tech. degree in Electronics and Communication Engineering from M.I.T Moradabad (India) and M.Tech. degree in Signal Processing and Digital Design from Delhi technological University, New Delhi, India. He worked as senio research scholar (SRF) in Delhi technological University. His research interests are signal processing and circuit estimation.

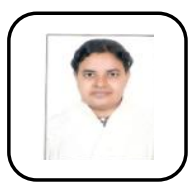

Sudipta Majumdar received B.Tech. and M.Tech degree from University of Allahabad. She received Ph.D. degree from Delhi University. She is working as Assistant Professor in Delhi Technological University from 2010. Her research interests include nonlinear analysis, signal processing and image processing.

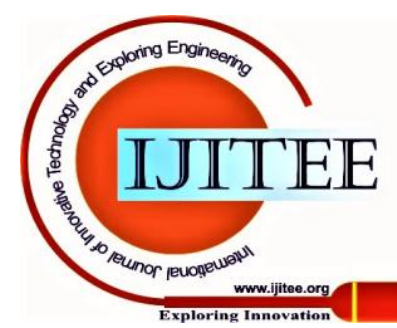

